\title{
ABUSO SEXUAL INFANTIL POR REPRESENTANTES DE LA IGLESIA CATÓLICA: EL CASO CHILENO
}

\section{Lorena Contreras Taibo ${ }^{a 1}\left(\mathbb{D}\right.$, Francisco Maffioletti Celedón $\left.{ }^{a}{ }^{(}\right), \&$ Noemí Pereda Beltrán}

Universidad Diego Portales, Santiago, Chile. ${ }^{\mathrm{a}}$

Universitat de Barcelona, Barcelona, España. ${ }^{\mathrm{b}}$

\section{RESUMEN}

La presente investigación describe las características de un grupo de 21 víctimas de abuso sexual por un representante de la Iglesia Católica en Chile. Se evaluaron las características de la victimización sexual y del victimario, la revelación, notificación y la vivencia de otras formas de victimización por personas no pertenecientes al clero. Los resultados muestran que los abusos se iniciaron en la pubertad, incluyeron contacto físico, fueron cometidos por varones, sacerdotes o párrocos, y fueron revelados por las víctimas en la edad adulta, notificando habitualmente a la propia Iglesia. La mayoría de víctimas también sufrieron malos tratos por parte de sus padres o cuidadores principales y abusos sexuales por parte de otras personas, lo que las convierte en polivíctimas y subraya la gravedad de estos casos.

\section{Palabras Claves}

abuso sexual; iglesia; infancia; Chile

\begin{abstract}
The present research aims to describe the characteristics of a group of 21 victims of sexual abuse by a representative of the Catholic Church in Chile. The characteristics of the sexual victimization and the perpetrator, the disclosure and notification, and the experience of other forms of victimization by nonclergy were evaluated. The results show that the abuses began at puberty, included behaviors with physical contact, were committed by men, mainly priests, and were revealed by the victims in adulthood, reported to the Church itself. Most victims also suffered maltreatment by their parents or primary caregivers and sexual abuse by other people, making them poly-victims and underscoring the seriousness of these cases.
\end{abstract}

\section{Keywords}

sexual abuse; church; childhood; Chile

\footnotetext{
${ }^{1}$ Correspondence about this article should be addressed to Lorena Contreras Taibo: lorena.contreras@udp.cl
} 


\section{CHILD SEXUAL ABUSE BY REPRESENTATIVES OF THE CATHOLIC CHURCH: THE CHILEAN CASE}

\section{Introducción}

La transgresión en el ámbito de la sexualidad constituye una grave forma de violencia, presente en todas las sociedades a lo largo del mundo. Así, los meta análisis señalan que cerca de un $19 \%$ de las mujeres y un $8-10 \%$ de los hombres sufren alguna forma de abuso sexual antes de cumplir la mayoría de edad (Barth, Bermetz, Heim, Trelle y Tonia, 2013; Pereda, Guilera, Forns, Gómez-Benito, 2009; Stoltenborgh, van IJzendoorn, Euser, y Bakermans-Kranenburg, 2011).

Con relación a los delitos sexuales, históricamente la preocupación se ha centrado en el abuso que se produce en el seno de la familia, o bien por parte de personas que pertenecen al entorno social inmediato. De este modo, el abuso sexual infantil que sucede en entornos religiosos ha cobrado relevancia recién durante los últimos quince años, jugando la prensa un rol central en la divulgación de estos casos (Denney, Kerley, y Gross, 2018). Investigaciones periodísticas como las del Boston Globe han permitido revelar fenómenos de abuso sistemático hacia niños, niñas y adolescentes producidos en contextos de iglesia.

Por su parte, en Chile son numerosos los casos de connotación pública que involucran hechos sistemáticos de abuso sexual infantil por parte de representantes de la iglesia, muchos de ellos sostenidos durante años o décadas. Entre ellos destacan los casos de reputados miembros del clero, como Fernando Karadima, Renato Poblete, John O`Reilly o Cristián Precht. Así, situaciones como las denunciadas en el Caso Maristas, Jesuitas o Legionarios de Cristo han sumido a congregaciones en escándalos mediáticos de proporciones. Tal ha sido la dimensión y el impacto que han provocado en la población chilena, que la Red de Sobrevivientes de Abuso Sexual Eclesiástico de Chile ha publicado el Mapa chileno de los delitos de abuso sexual y de conciencia cometidos en entornos eclesiásticos ${ }^{2}$, que detalla por región los casos registrados.

Como resultado de los reportes efectuados en distintos lugares del mundo, académicos y comisiones nombradas para este fin han comenzado estudios en diversos países como Australia, Austria, Bélgica, Inglaterra y Gales, Irlanda, Países Bajos y El

\footnotetext{
${ }^{2}$ Véase https://www.redsobrevivientes.org/post/mapa-abusos
} 
Vaticano (Terry, 2015), creciendo de modo relevante el cuerpo de investigación disponible a lo largo del mundo.

Respecto a la magnitud de este fenómeno, el estudio de Langeland, Hoogendoorn, Mager, Smit, y Dajier (2015) señala que el 1,7\% de los encuestados reportó abuso sexual infantil por parte de un representante de la iglesia católica. Los abusos fueron 5 veces más prevalentes entre quienes recibieron educación católica, resultando también mayor su frecuencia en sujetos de edad más avanzada y en quienes estuvieron institucionalizados (Langeland et al., 2015).

Por su parte, de acuerdo a la información aportada por John Jay College of Criminal Justice (2004), entre el 4,0\% y el 4,6\% de todos los sacerdotes activos entre 1950 y 2002 tuvieron acusaciones de abuso. Por cierto, la mera acusación no implica una prueba de culpabilidad, sin embargo, es necesario tener presente que existe una importante proporción de cifra negra en delitos sexuales, cercana al 90\% de casos que nunca llegan a denunciarse a las autoridades (Azócar y Undurraga, 2005).

Respecto a figura del autor, la evidencia disponible señala que menos de un 5\% presentan conductas compatibles con pedofilia, tampoco resultan más prevalentes que en otros sacerdotes los desórdenes de personalidad o trastornos del ánimo (Terry et al., 2011). Por su parte, Calkins, Fargo, Jeglic, y Terry (2015) señalan que su propia historia de abuso sexual se erigió como el principal factor de riesgo para la perpetración de abusos. Junto con esto, presentan mayores probabilidades de trabajar con jóvenes, pues seguramente orientan su labor en esa dirección (Calkins et al., 2015).

Respecto a la caracterización de sus víctimas, si bien en población general el abuso sexual infantil es mucho más frecuente en personas de sexo femenino, esta proporción se invierte cuando se trata de delitos sexuales en contexto de iglesia. De este modo, si es que en el abuso sexual infantil en población general se arriba a una proporción de un varón por cada tres mujeres (Barth et al., 2013; Echeburúa, y Guerricaechevarría, 2008; Ministerio del Interior, 2013; Madrid, 2015), en el abuso eclesiástico se registran cuatro víctimas varones por cada mujer (John Jay College of Criminal Justice, 2004; Langeland et al., 2015).

Asimismo, las edades de mayor riesgo también se modifican. En el abuso sexual infantil en población general la edad preescolar y la pubertad son edades de mayor exposición fundamentalmente en mujeres. En el caso de los varones, la mayor frecuencia se observa en preescolares, luego de lo cual el riesgo decrece paulatinamente con el paso de los años (Contreras, Huepe y Navarrete, 2020). No obstante, en el contexto eclesiástico 
la mayoría de las víctimas son varones en etapa de adolescencia (John Jay College of Criminal Justice, 2004).

El abuso sexual infantil resulta particularmente frecuente $(68 \%)$ entre quienes vivieron alguna forma de violencia por parte de representantes de la iglesia (LuegerSchuster et al., 2014); padeciendo muchos de ellos formas de abuso en extremo intrusivas. Concordantemente con lo anterior, Denney et al. (2018) refieren en el 80\% de los casos delitos de contacto directo y físico hacia la víctima. De manera similar, Rassenhofer, Zimmer, Spröber, y Fegert (2015) reportan que un 73,1\% de hechos implican contacto o penetración. La mayor parte de los abusos reportados por las víctimas $(88,6 \%)$ son de carácter reiterado (Rassenhofer et al., 2015).

De acuerdo a Lueger-Schuster et al. (2014), el 81,7\% de los delitos sexuales vinculados a la iglesia ocurrieron en contextos donde residen los niños, niñas y adolescentes, o bien el clero, tales como internados, orfanatos, monasterios o conventos; mientras el 14,4\% ocurrieron en lugares de culto, como parroquias o iglesias. Denney et al. (2018), además de estas locaciones, identifican sitios externos y el hogar de la propia víctima. Por su parte, Rassenhofer et al. (2015) señalan que la mayoría de estos hechos $(66,8 \%)$ se produjeron en actividades congregacionales.

Un hallazgo particularmente preocupante radica en la duración media del contacto entre el afectado y el autor, el cual asciende a 4,8 años (Lueger-Schuster et al., 2014). Durante todo este tiempo la víctima suele permanecer silenciada y atrapada en una relación traumática de la cual difícilmente puede escapar, con el daño psíquico que ello conlleva.

De hecho, el $45 \%$ de las víctimas refiere problemas psicosociales reactivos al abuso sexual en entornos eclesiásticos (Rassenhofer et al., 2015). Respecto a la naturaleza de este daño, Lueger-Schuster et al. (2014) han identificado la presencia de TEPT en el $48,6 \%$ de los adultos sobrevivientes.

Desde la perspectiva de la víctima, la investigación da cuenta de vulnerabilidad, shock, así como sentimientos de vergüenza, culpa e impotencia. Todo ello es potenciado por la reacción de la propia iglesia, generando en los sobrevivientes una sensación de traición a la confianza religiosa, dado que su reacción es percibida como negadora y minimizadora de los abusos, manteniendo la victimización en la esfera de lo privado (Varona y Martínez, 2015).

Las investigaciones empíricas que abordan el abuso sexual infantil en contextos eclesiásticos han sido desarrolladas en países del primer mundo, tales como EEUU, 
Australia, Países Bajos, Austria y Alemania. Así, resulta notable la ausencia de estudios empíricos desarrollados en América Latina y en otros países en vías de desarrollo en miras a caracterizar este fenómeno, lo cual genera interrogantes acerca de la aplicabilidad de las conclusiones a contextos muy distintos.

Dada la escasez de publicaciones destinadas a caracterizar el abuso sexual infantil ocurrida en el entorno eclesiástico, y particularmente en relación a la carencia de estudios en la realidad latinoamericana o chilena, la presente investigación tiene por objetivo describir las principales características de la victimización sexual contra niños, niñas y adolescentes, cometida por parte de representantes de la iglesia católica en Chile. Todo ello, a partir de la información aportada por los propios sobrevivientes.

\section{Método}

\section{Tipo de estudio}

Se ha desarrollado un estudio cuantitativo con un alcance exploratoriodescriptivo, en tanto se pretende conocer cómo se presenta la problemática del abuso sexual infantil perpetrado por representantes de la iglesia católica, aspecto que, hasta donde tenemos conocimiento, a la fecha no ha sido estudiado empíricamente en Chile ni en Latinoamérica.

Así, este estudio no experimental de tipo transeccional pretende dar cuenta de las características de las víctimas, los perpetradores, así como las situaciones de victimización originadas en este contexto.

\section{Participantes}

Las características de la muestra hacen difícil el acceso a ella, pues hablamos de personas adultas que han sido víctima de abuso sexual infantil, en cualquiera de sus tipos penales, por parte de personas pertenecientes al entorno eclesiástico. Por tanto, se utilizó un muestreo no probabilístico por conveniencia, mediante la publicación del enlace de la encuesta en redes sociales y a través de su difusión a las asociaciones de la sociedad civil que trabajan con víctimas de abuso sexual en el país. Asimismo, se solicitó el apoyo de sobrevivientes que han hecho pública su victimización para ayudar a difundir la encuesta. Como resultado, se encuestó a un total de 21 adultos, de los cuales 11 fueron mujeres $(52,4 \%)$ y 10 varones $(47,6 \%)$, con un rango de edad que va de 21 a 55 años, con una 
media de 39,5 años (mediana=41; DT $=9,5)$. De estos, un 90,5\% corresponden a ciudadanos chilenos, y el otro 9,5\% son originarios de otros países de América Latina.

En cuanto a su nivel de estudios, un $42,9 \%$ poseen estudios universitarios o grados equivalentes, a lo cual se suma un 42,9\% adicional que refiere estudios de Máster o postgrado. Por su parte un 9,5\% del total refiere estudios superiores técnicos o equivalentes, existiendo un solo caso con estudios de educación media. Así, un 85,8\% de quienes han respondido el cuestionario presentan estudios de grado universitario o superior.

En cuanto a la situación laboral, la amplia mayoría (81\%) señala trabajar como "asalariado", lo que en el caso de los hombres asciende al 100\%, mientras que sólo el $63,6 \%$ de las mujeres trabaja en esta condición. A esto le sigue la categoría de "trabajador por cuenta propia" (19\%). Las demás categorías (estudiante; cuidado de casa, hijos o familiar; prefiere no contestar) representan cada una de ellas un 4,8\%.

Con respecto del estado civil de los encuestados, más de la mitad de las mujeres están solteras $(54,5 \%)$, seguidas por quienes se encuentran en pareja $(27,3 \%)$ y finalmente quienes han concluido una relación (18,2\%). En cambio, la mayoría de los varones está en pareja (60\%), siendo igual el porcentaje que está soltero (20\%) y que ha finalizado una relación $(20 \%)$.

Sobre la orientación sexual de los encuestados, un 61,9\% señala ser heterosexual, correspondiendo al 72,7\% de las mujeres y al 50\% de los hombres. Asimismo, un 28,5\% manifiesta una orientación homosexual, un 4,8\% bisexual, y el mismo porcentaje prefiere "no contestar".

En cuento a su creencia religiosa actual, dos tercios de los encuestados $(66,7 \%)$ mantienen una creencia religiosa ( $80 \%$ de los hombres y 54,5\% de las mujeres), mientras un $28,6 \%$ de los participantes no la tienen, y en un $4,7 \%$ prefiere no contestar (Ver Tabla $1)$.

\section{Instrumentos}

Para el presente estudio, el equipo de investigación generó una batería de preguntas, basada en el análisis de publicaciones previas sobre esta problemática, organizada en base a siete secciones. 
Tabla 1.

Características sociodemográficas

\begin{tabular}{|c|c|c|c|c|c|c|}
\hline \multirow[t]{2}{*}{ Variable } & \multicolumn{2}{|c|}{$\begin{array}{l}\text { Hombre } \\
(n=10)\end{array}$} & \multicolumn{2}{|c|}{$\begin{array}{l}\text { Mujer } \\
(n=11)\end{array}$} & \multicolumn{2}{|c|}{$\begin{array}{c}\text { Total } \\
(n=21)\end{array}$} \\
\hline & $n$ & $\%$ & $n$ & $\%$ & $n$ & $\%$ \\
\hline \multicolumn{7}{|l|}{ País de Origen } \\
\hline Chile & 8 & $80,0 \%$ & 11 & $100,0 \%$ & 19 & $90,5 \%$ \\
\hline Otro (América del Sur) & 2 & $20,0 \%$ & 0 & $0,0 \%$ & 2 & $9,5 \%$ \\
\hline \multicolumn{7}{|l|}{ Nivel de Estudios } \\
\hline Educación Media & 1 & $10,0 \%$ & 0 & $0,0 \%$ & 1 & $4,7 \%$ \\
\hline Grado técnico o equivalente & 1 & $10,0 \%$ & 1 & $9,1 \%$ & 2 & $9,5 \%$ \\
\hline $\begin{array}{l}\text { Grado universitario o } \\
\text { equivalente }\end{array}$ & 5 & $50,0 \%$ & 4 & $36,4 \%$ & 9 & $42,9 \%$ \\
\hline $\begin{array}{l}\text { Máster, postgrado o } \\
\text { doctorado }\end{array}$ & 3 & $30,0 \%$ & 6 & $54,5 \%$ & 9 & $42,9 \%$ \\
\hline \multicolumn{7}{|l|}{ Situación Laboral* } \\
\hline Estudiante & 0 & $0,0 \%$ & 1 & $9,1 \%$ & 1 & $4,8 \%$ \\
\hline $\begin{array}{l}\text { Cuidado de casa, hijos o } \\
\text { familiar }\end{array}$ & 0 & $0,0 \%$ & 1 & $9,1 \%$ & 1 & $4,8 \%$ \\
\hline Trabaja asalariado & 10 & $100 \%$ & 7 & $63,6 \%$ & 17 & $81,0 \%$ \\
\hline Trabaja por cuenta propia & 1 & $10 \%$ & 3 & $27,3 \%$ & 4 & $19,0 \%$ \\
\hline Prefiere no contestar & 0 & $0,0 \%$ & 1 & $9,1 \%$ & 1 & $4,8 \%$ \\
\hline \multicolumn{7}{|l|}{ Estado civil } \\
\hline Soltero/a & 2 & $20,0 \%$ & 6 & $54,5 \%$ & 8 & $38,1 \%$ \\
\hline Casado/a & 2 & $20,0 \%$ & 2 & $18,2 \%$ & 4 & $19,0 \%$ \\
\hline Con pareja estable & 4 & $40,0 \%$ & 1 & $9,1 \%$ & 5 & $23,8 \%$ \\
\hline Separado/a & 1 & $10,0 \%$ & 2 & $18,2 \%$ & 3 & $14,3 \%$ \\
\hline Divorciado/a & 1 & $10,0 \%$ & 0 & $0,0 \%$ & 1 & $4,8 \%$ \\
\hline \multicolumn{7}{|l|}{ Orientación sexual } \\
\hline Heterosexual & 5 & $50,0 \%$ & 8 & $72,7 \%$ & 13 & $61,9 \%$ \\
\hline Homosexual & 4 & $40,0 \%$ & 2 & $18,2 \%$ & 6 & $28,5 \%$ \\
\hline Bisexual & 0 & $0,0 \%$ & 1 & $9,1 \%$ & 1 & $4,8 \%$ \\
\hline Prefiero no contestar & 1 & $10,0 \%$ & 0 & $0,0 \%$ & 1 & $4,8 \%$ \\
\hline
\end{tabular}

* Permite más de una categoría de respuesta

A continuación, se detallan las variables consideradas en cada una de las secciones abordadas en el presente artículo:

(1) Información personal de carácter general acerca de los participantes: sexo; edad; lugar de nacimiento; estado civil; orientación sexual; nivel de estudios; situación laboral. Aquí se incorporan también preguntas relativas a su fe, religión y sistema de creencias, tales como: religión; asistencia a oficios religiosos; fortaleza y consuelo asociada a la religión; y presencia de fe.

(2) Información relativa a la temática de victimización sexual por parte de la Iglesia: existencia de victimización sexual infantil por parte de un representante de la iglesia católica; edad de inicio y término; tipo de victimización sexual; 
número de perpetradores; sexo de perpetradores; cargo de perpetradores; utilización de símbolos, imágenes u objetos religiosos durante los abusos; utilización de creencias religiosas para la perpetración de abusos; efectos de los abusos en la fe; primera revelación de abusos; edad al momento de la develación; destinatarios de la develación; credibilidad en el relato de los abusos; revelaciones posteriores; respuestas de apoyo; notificación a las autoridades; e impacto en el bienestar emocional.

(3) Formas de victimización sexual cometidas por otras personas no vinculadas a la iglesia: existencia de abuso sexual infantil por personas que no representan a la iglesia católica; edad de inicio y fin; tipo de victimización sexual; número de perpetradores; sexo de perpetradores; edad perpetradores; relación con la víctima; efecto del abuso en la fe; revelación inicial de los abusos; edad de develación; destinatario de la develación; credibilidad en su relato; revelaciones posteriores; notificación a la autoridad; impacto sobre el bienestar emocional.

(4) Otras formas de victimización hacia la víctima perpetradas por sus padres o cuidadores principales: existencia de maltrato físico; existencia de maltrato psicológico; presencia de manipulación parental; presencia de negligencia parental o por cuidadores; relación temporal de estas formas de violencia con el abuso eclesiástico.

(5) Consecuencias psicológicas de la victimización (como trastornos ansiosos, depresivos, problemas de sueño, entre otros).

(6) Información acerca de la espiritualidad de la víctima (sentido de la vida, pensamientos sobre la muerte, entre otros).

(7) Adaptación española del Posttraumatic Growth Inventory (PTGI, Tedeschi y Calhoun, 1996).

Los tres últimos aspectos no han sido objeto de estudio en el presente artículo, si bien se analizarán en publicaciones posteriores.

\section{Procedimiento}

Para acceder a los participantes del estudio, se utilizó un muestreo no probabilístico por conveniencia y se comenzó por identificar las organizaciones que trabajan con víctimas de abuso sexual en el territorio nacional, así como aquellas que trabajan con sobrevivientes de abuso sexual eclesiástico, contactándolas para solicitar su 
colaboración. De este modo, se pidió a dichas organizaciones que pudieran difundir el link de la encuesta entre potenciales participantes, y que pudieran incluir este link en sus páginas web. Junto con esto, se contactó a víctimas que han hecho públicos los abusos que habían sufrido por parte de representantes de la iglesia para ayudar a difundir esta encuesta entre sus contactos. Asimismo, la encuesta fue difundida a través de redes sociales, de manera de abarcar la mayor cantidad de población.

Este estudio ha seguido los principios éticos de la Declaración de Helsinki (AMM, 2013) y el Código de Ética Profesional del Colegio de Psicólogos de Chile (Colegio de Psicólogos de Chile, 1999), siendo aprobado por la Comisión de Bioética de la Universidad de Barcelona (IRB00003099), en la cual radica esta investigación.

\section{Análisis estadísticos}

Se utilizó el paquete estadístico SPSS Statistics, en su versión 26. En relación a los objetivos del estudio, primeramente se calcularon los estadísticos descriptivos tendientes a determinar las características sociodemográficas de la muestra estudiada. A continuación, y teniendo como variable de segmentación de la muestra el sexo de los participantes, se efectuó un análisis descriptivo destinado a caracterizar el abuso sexual vivenciado en contexto eclesiástico durante su infancia o adolescencia, así como las características del victimario, la revelación de estos hechos y la notificación a las autoridades. Junto con esto, se analizan otras formas de victimización experimentadas por los sobrevivientes, dando cuenta de la relación temporal de estos hechos con la victimización vivida en contexto eclesiástico.

\section{Resultados}

En la muestra estudiada $(n=21)$, la edad de inicio del abuso sexual infantil en el contexto eclesiástico tiene una media de 12,1 años (mediana=13; DT=4,2); finalizando en promedio a los 13,7 años (mediana=15; DT=5,0). Respecto a la duración del abuso, este va desde situaciones puntuales hasta abusos sostenidos durante 6 años, con una media de 1,5 años (mediana=1; DT=1,9).

Respecto a las características del abuso sexual infantil perpetrado por representantes de la iglesia católica (ver tabla 2), el 66,7\% $(n=14)$ refieren haber sufrido una victimización sexual "con contacto" (ej: tocaciones, masturbación), mientras el $33,3 \%(n=7)$ manifiestan que hubo "introducción de objeto o parte del cuerpo" del 
perpetrador en ano, vagina o boca de la víctima. Por su parte, sólo un 14,3\% ( $\mathrm{n}=3)$ manifiesta que la victimización sexual se realizó "sin contacto". Cabe hacer presente que los encuestados pueden haber respondido afirmativamente en más de una categoría, por lo cual la suma de los porcentajes supera el $100 \%$.

El 28,6\% (n=6) de los sobrevivientes reconocen la utilización de algún objeto, símbolo o imagen religiosa en la perpetración de los abusos, entre los cuales mencionan el cáliz, altar, crucifico, sagrario, oración y utilización de la oficina parroquial. Asimismo, en el 71,4\% (n=15) de los casos los sobrevivientes consideran que el abusador utilizó sus creencias religiosas para llevar a cabo los abusos. De este modo, refieren alusiones directas a Dios en el marco de las estrategias de victimización desplegadas, en las cuales se insinúa una aceptación divina, como por ejemplo: "Dios así lo quiere", "Dios lo entiende", "Eres un elegido de Dios". Junto con esto, se reportan verbalizaciones que culpabilizan a la propia víctima del abuso sexual: "Eres un pecador y Dios te perdona", "Tienes que rezar porque me hiciste pecar" (Ver Tabla 2).

Tabla 2.

Características del abuso sexual infantil

\begin{tabular}{|c|c|c|c|c|c|c|}
\hline \multirow[b]{2}{*}{ Variable } & \multicolumn{2}{|c|}{$\begin{array}{l}\text { Hombre } \\
(n=10)\end{array}$} & \multicolumn{2}{|c|}{$\begin{array}{l}\text { Mujer } \\
(n=11)\end{array}$} & \multicolumn{2}{|c|}{$\begin{array}{c}\text { Total } \\
(n=21)\end{array}$} \\
\hline & $n$ & $\%$ & $n$ & $\%$ & $n$ & $\%$ \\
\hline \multicolumn{7}{|l|}{ Tipo victimización sexual* } \\
\hline Sin contacto & 2 & $20,0 \%$ & 1 & $9,1 \%$ & 3 & $14,3 \%$ \\
\hline Con contacto & 7 & $70,0 \%$ & 7 & $63,6 \%$ & 14 & $66,7 \%$ \\
\hline $\begin{array}{l}\text { Introducción objeto o parte de } \\
\text { victimario a víctima }\end{array}$ & 3 & $30,0 \%$ & 4 & $36,4 \%$ & 7 & $33,3 \%$ \\
\hline \multicolumn{7}{|l|}{ Duración del abuso sexual } \\
\hline Menos de 1 año & 5 & $50,0 \%$ & 5 & $45,5 \%$ & 10 & $47,6 \%$ \\
\hline 1 año & 2 & $20,0 \%$ & 1 & $9,1 \%$ & 3 & $14,3 \%$ \\
\hline 2 años & 2 & $20,0 \%$ & 1 & $9,1 \%$ & 3 & $14,3 \%$ \\
\hline 3 años & 0 & $0,0 \%$ & 1 & $9,1 \%$ & 1 & $4,8 \%$ \\
\hline 4 años & 1 & $10,0 \%$ & 1 & $9,1 \%$ & 2 & $9,5 \%$ \\
\hline 5 años & 0 & $0,0 \%$ & 1 & $9,1 \%$ & 1 & $4,8 \%$ \\
\hline 6 años & 0 & $0,0 \%$ & 1 & $9,1 \%$ & 1 & $4,8 \%$ \\
\hline \multicolumn{7}{|l|}{ Objeto, símbolo o imagen religiosa } \\
\hline Sí & 3 & $30,0 \%$ & 3 & $27,3 \%$ & 6 & $28,6 \%$ \\
\hline No & 6 & $60,0 \%$ & 5 & $45,5 \%$ & 11 & $52,4 \%$ \\
\hline No lo recuerdo & 1 & $10,0 \%$ & 3 & $27,3 \%$ & 4 & $19,0 \%$ \\
\hline \multicolumn{7}{|l|}{ Creencia religiosa } \\
\hline Sí & 8 & $80,0 \%$ & 6 & $54,5 \%$ & 14 & $66,7 \%$ \\
\hline No & 2 & $20,0 \%$ & 4 & $36,4 \%$ & 6 & $28,6 \%$ \\
\hline Prefiero no contestar & 0 & $0,0 \%$ & 1 & $9,1 \%$ & 1 & $4,7 \%$ \\
\hline
\end{tabular}

* Permite más de una categoría de respuesta

Ahora bien, con relación a las características del victimario, los encuestados reportan entre 1 y 6 perpetradores, con una media de 1,5 autores (mediana=1; DT=1,2). 
En un 76,2\% ( $n=16)$ del total de casos las victimizaciones sexuales fueron cometidas por un solo victimario ( $81,8 \%$ en mujeres y $70 \%$ en varones). Los varones reportan en un $100 \%$ de los casos $(\mathrm{n}=10)$ que sus victimarios fueron hombres, mientras las mujeres refieren victimarios hombres en el 90,9\% de los casos $(n=10)$. Estos datos relevan que un 95,2\% ( $\mathrm{n}=20)$ de las victimizaciones sexuales, tanto contra hombres como mujeres, fueron cometidas por victimarios hombres.

Respecto a la función ejercida por el perpetrador, un $52,4 \% \quad(n=11)$ de las victimizaciones sexuales fueron cometidas por "sacerdotes o párrocos", un $19 \%(n=4)$ por laicos, un 19\% $(\mathrm{n}=4)$ por "consagrados" (monjes/as, abad/esa, fraile, hermano/a), y un $14,3 \%(n=3)$ por "otros" (Ver Tabla 3$)$.

Tabla 3.

Características del victimario

\begin{tabular}{|c|c|c|c|c|c|c|}
\hline \multirow[t]{2}{*}{ Variable } & \multicolumn{2}{|c|}{$\begin{array}{c}\text { Hombres } \\
(n=10)\end{array}$} & \multicolumn{2}{|c|}{$\begin{array}{l}\text { Mujeres } \\
(n=11)\end{array}$} & \multicolumn{2}{|c|}{$\begin{array}{c}\text { Total } \\
(n=21)\end{array}$} \\
\hline & $n$ & $\%$ & $n$ & $\%$ & $n$ & $\%$ \\
\hline \multicolumn{7}{|l|}{ Número victimarios } \\
\hline 1 & 7 & $70,0 \%$ & 9 & $81,8 \%$ & 16 & $76,2 \%$ \\
\hline 2 & 1 & $10,0 \%$ & 1 & $9,1 \%$ & 2 & $9,5 \%$ \\
\hline 3 & 1 & $10,0 \%$ & 1 & $9,1 \%$ & 2 & $9,5 \%$ \\
\hline Múltiples & 1 & $10,0 \%$ & 0 & $0 \%$ & 1 & $4,8 \%$ \\
\hline \multicolumn{7}{|l|}{ Sexo victimario } \\
\hline Hombre & 10 & $100,0 \%$ & 10 & $90,9 \%$ & 20 & $95,2 \%$ \\
\hline Mujer & 0 & $0,0 \%$ & 1 & $9,1 \%$ & 1 & $4,8 \%$ \\
\hline \multicolumn{7}{|l|}{ Cargo religioso* } \\
\hline Laico & 3 & $30,0 \%$ & 1 & $9,1 \%$ & 4 & $19,0 \%$ \\
\hline Consagrado & 2 & $20,0 \%$ & 2 & $18,2 \%$ & 4 & $19,0 \%$ \\
\hline Sacerdote, párroco & 5 & $50,0 \%$ & 6 & $54,5 \%$ & 11 & $52,4 \%$ \\
\hline Otros & 1 & $10,0 \%$ & 2 & $18,2 \%$ & 3 & $14,3 \%$ \\
\hline
\end{tabular}

* Permite más de una categoría de respuesta

Los resultados indican que en un $81 \%$ de los casos $(n=17)$ se produjo una revelación del abuso sexual sufrido en contexto eclesiástico, lo cual resulta más prevalente en mujeres $(90,9 \% ; n=10)$ que en varones $(70 \% ; n=7)$. En promedio, esta primera revelación se produce a los 28,4 años (mediana=30; DT=13,8), es decir 16,3 años después de la media de inicio del abuso.

Los destinatarios más frecuentes de la primera revelación de estos hechos fueron su "pareja" $(35,3 \% ; n=6)$, la "madre" $(29,4 \% ; n=5)$, un "amiga/o" $(23,5 \% ; n=4)$, y su "padre" $(17,6 \% ; n=3)$. Menos frecuente resultó la revelación a "otro familiar", a un 
"profesional", o a un "representante de la iglesia católica" (11,8\% en los tres casos). Por su parte, sólo un encuestado (5,9\%) contestó que develó ante un “desconocido", e idéntico porcentaje lo hizo ante "otro".

De aquellos que revelaron el abuso sexual vivido $(\mathrm{n}=17)$, más de la mitad $(52,9 \%$; $\mathrm{n}=9$ ) considera que todos o casi todos creyeron en su relato, mientras que una cifra algo menor $(41,2 \% ; n=7)$ evalúa que algunos creyeron y otros no, restando solo un participante $(5,9 \%)$ que no se sintió creído. Entre quienes develaron, el 76,5\% (n=13) sintió una respuesta de apoyo, mientras un $23,5 \%(n=4)$ considera lo contrario. Luego de esta revelación inicial, sólo 1 participante de sexo femenino volvió a explicarlo más adelante.

Respecto de la "notificación” de esta situación de victimización, el 47,6\% ( $n=10)$ de los encuestados refiere haber puesto en conocimiento de los hechos a las autoridades. La gran mayoría $(80 \% ; n=8)$ lo hizo ante un "representante de la Iglesia Católica", la mitad $(50 \% ; n=5)$ ante el "Ministerio Público", y un tercio $(30 \% ; n=3)$ ante el "Poder Judicial". Esta preeminencia de la notificación ante autoridades eclesiásticas es más pronunciada en varones $(85,7 \%)$ que en mujeres $(66,7 \%)$. Dichas notificaciones fueron efectuadas en un $60 \%(n=6)$ de los casos por la propia persona afectada $(71,4 \%$ de los hombres y 33,3\% de las mujeres), en un $40 \%(n=4)$ de los casos por uno de sus padres, en un $20 \%(\mathrm{n}=2)$ otro familiar, y en un caso $(10 \%)$ a un profesional. Este contacto con la autoridad derivado de la notificación tuvo un impacto medio para los sobrevivientes, situándose en una media de 4,1 y una mediana de 3,5 (mínimo=0, máximo 10, DT=3,8), más cercano al polo negativo (Ver Tabla 4).

Además del abuso sexual infantil por representantes de la iglesia, 17 de los 21 encuestados $(81 \%)$ reportaron haber vivido otras formas de victimización, con una media de 3,1 tipos de violencia (DT=2,1, mínimo=1, máximo=9). Así, dan cuenta de "maltrato emocional por cuidadores" $(57,1 \% ; n=12)$, "maltrato físico por cuidadores" $(52,4 \%$; $\mathrm{n}=11)$ "abuso sexual no eclesiástico" $(52,4 \% ; n=11)$, "manipulación parental” $(23,8 \%$; n=5) y "negligencia" $(33,3 \% ; n=7)$. Cabe hacer presente que, a excepción de la "manipulación parental", más prevalente en varones, todas las otras formas de victimización resultan más frecuentes en mujeres, cifra que llega a más del doble en caso de delitos sexuales en contextos no eclesiásticos (Ver Tabla 5). 
Tabla 4.

Características de la primera revelación y notificación del abuso sexual infantil

\begin{tabular}{|c|c|c|c|c|c|c|}
\hline \multirow[t]{2}{*}{ Variable } & \multicolumn{2}{|c|}{$\begin{array}{c}\text { Hombres } \\
(n=10)\end{array}$} & \multicolumn{2}{|c|}{$\begin{array}{c}\text { Mujeres } \\
(n=11)\end{array}$} & \multicolumn{2}{|c|}{$\begin{array}{c}\text { Total } \\
(n=21)\end{array}$} \\
\hline & $n$ & $\%$ & $n$ & $\%$ & $n$ & $\%$ \\
\hline \multicolumn{7}{|l|}{ Revelación del ASI } \\
\hline $\mathrm{Si}$ & 7 & $70,0 \%$ & 10 & $90,9 \%$ & 17 & $81,0 \%$ \\
\hline No & 3 & $30,0 \%$ & 1 & $9,1 \%$ & 4 & $19,0 \%$ \\
\hline \multicolumn{7}{|l|}{ Persona a quien se reveló* } \\
\hline Madre & 2 & $28,6 \%$ & 3 & $30,0 \%$ & 5 & $29,4 \%$ \\
\hline Padre & 1 & $14,3 \%$ & 2 & $20,0 \%$ & 3 & $17,6 \%$ \\
\hline Otro familiar & 1 & $14,3 \%$ & 1 & $10,0 \%$ & 2 & $11,8 \%$ \\
\hline Amigo/a & 2 & $28,6 \%$ & 2 & $20,0 \%$ & 4 & $23,5 \%$ \\
\hline Pareja & 4 & $57,1 \%$ & 2 & $20,0 \%$ & 6 & $35,3 \%$ \\
\hline Profesional & 0 & $0,0 \%$ & 2 & $20,0 \%$ & 2 & $11,8 \%$ \\
\hline Desconocido/a & 0 & $0,0 \%$ & 1 & $10,0 \%$ & 1 & $5,9 \%$ \\
\hline Representante Iglesia Católica & 1 & $14,3 \%$ & 1 & $10,0 \%$ & 2 & $11,8 \%$ \\
\hline Otro & 0 & $0,0 \%$ & 1 & $10,0 \%$ & 1 & $5,9 \%$ \\
\hline \multicolumn{7}{|l|}{ Notificación* } \\
\hline Sí & 7 & & 3 & & 10 & \\
\hline Representante Iglesia Católica & 6 & $85,7 \%$ & 2 & $66,7 \%$ & 8 & $80,0 \%$ \\
\hline Poder Judicial & 2 & $28,6 \%$ & 1 & $33,3 \%$ & 3 & $30,0 \%$ \\
\hline Ministerio Público & 4 & $57,1 \%$ & 1 & $33,3 \%$ & 5 & $50,0 \%$ \\
\hline \multicolumn{7}{|l|}{ Persona que notificó* } \\
\hline Él/ella mismo/a & 5 & $71,4 \%$ & 1 & $33,3 \%$ & 6 & $60,0 \%$ \\
\hline Madre & 1 & $14,3 \%$ & 2 & $66,7 \%$ & 3 & $30,0 \%$ \\
\hline Padre & 0 & $0,0 \%$ & 1 & $33,3 \%$ & 1 & $10,0 \%$ \\
\hline Otro familiar & 0 & $0,0 \%$ & 2 & $66,7 \%$ & 2 & $20,0 \%$ \\
\hline Profesional & 1 & $14,3 \%$ & 0 & $0,0 \%$ & 1 & $10,0 \%$ \\
\hline Representante Iglesia Católica & 0 & $0,0 \%$ & 0 & $0,0 \%$ & 0 & $0,0 \%$ \\
\hline
\end{tabular}

* Permite más de una categoría de respuesta

Tabla 5.

Otras experiencias de victimización

\begin{tabular}{|c|c|c|c|c|c|c|}
\hline \multirow[t]{2}{*}{ Variable } & \multicolumn{2}{|c|}{$\begin{array}{c}\text { Hombres } \\
(n=10)\end{array}$} & \multicolumn{2}{|c|}{$\begin{array}{l}\text { Mujeres } \\
(n=11)\end{array}$} & \multicolumn{2}{|c|}{$\begin{array}{c}\text { Total } \\
(n=21)\end{array}$} \\
\hline & $n$ & $\%$ & $n$ & $\%$ & $n$ & $\%$ \\
\hline Abuso sexual no eclesiástico & 3 & $30,0 \%$ & 8 & $72,7 \%$ & 11 & $52,4 \%$ \\
\hline Maltrato físico por cuidadores & 4 & $40,0 \%$ & 7 & $63,6 \%$ & 11 & $52,4 \%$ \\
\hline Maltrato emocional por cuidadores & 5 & $50,0 \%$ & 7 & $63,6 \%$ & 12 & $57,1 \%$ \\
\hline Manipulación parental & 3 & $30,0 \%$ & 2 & $18,2 \%$ & 5 & $23,8 \%$ \\
\hline Negligencia & 2 & $20,0 \%$ & 5 & $45,5 \%$ & 7 & $33,3 \%$ \\
\hline
\end{tabular}


En relación a estas otras formas de victimización, resulta relevante analizar la dimensión temporal para comprender cómo estas anteceden, acompañan o se presentan con posterioridad al abuso eclesiástico. En este punto, y tal como muestra la tabla 6, las diferentes formas de victimización se presentan, en términos generales, tanto antes, como durante y con posterioridad al abuso eclesiástico. Sin embargo, la manipulación parental suele anteceder a este tipo de abusos, en los casos en que se presenta (Ver Tabla 6).

Tabla 6.

Otras experiencias de victimización en relación con el abuso sexual infantil por representantes de la iglesia católica

\begin{tabular}{|c|c|c|c|c|c|c|c|}
\hline \multirow[t]{2}{*}{ Variable* } & \multicolumn{2}{|c|}{ Antes } & \multicolumn{2}{|c|}{ Mientras } & \multicolumn{2}{|c|}{ Después } & \multirow{2}{*}{$\frac{\text { Total }}{n}$} \\
\hline & $n$ & $\%$ & $n$ & $\%$ & $n$ & $\%$ & \\
\hline Abuso sexual & 5 & $45,5 \%$ & 2 & $18,2 \%$ & 4 & $36,4 \%$ & 11 \\
\hline Maltrato físico & 6 & $54,5 \%$ & 5 & $45,5 \%$ & 6 & $54,5 \%$ & 11 \\
\hline Maltrato emocional & 6 & $50,0 \%$ & 5 & $41,7 \%$ & 7 & $58,3 \%$ & 12 \\
\hline Manipulación parental & 3 & $60,0 \%$ & 1 & $20,0 \%$ & 1 & $20,0 \%$ & 5 \\
\hline Negligencia & 4 & $57,1 \%$ & 4 & $57,1 \%$ & 5 & $71,4 \%$ & 7 \\
\hline
\end{tabular}

* Estas experiencias de victimización pueden haber sucedido antes, mientras y/o después del abuso sexual eclesiástico.

\section{Discusión}

El abuso sexual infantil en contexto eclesiástico constituye un tema silenciado por décadas en América Latina, territorio en el cual desde tiempos de la colonia el vínculo con la iglesia ha sido tradicionalmente importante. Si bien el trabajo de los medios de comunicación y de los sobrevivientes han permitido visibilizar este fenómeno y su gravedad, llama la atención la escasez de estudios empíricos que aborden el tema, tanto en Chile como en Latinoamérica.

Entendemos que, en todo el mundo, acceder a los sobrevivientes de abuso sexual eclesiástico con fines de investigación es en extremo difícil, tal como lo señalan estudios de revisión sobre el tema (Böhm, Zollner, Fegert y Liebhardt, 2014). Esta dificultad, sin embargo, es aún mayor en un país de tradición católica como es Chile, donde la iglesia y el poder político han estado estrechamente interrelacionados (Cancino, 2001). El malestar y desconfianza generados por la experiencia de abuso sexual también puede influir en el rechazo hacia los investigadores y hacia la investigación (McLaughlin, 1994). A su vez, en otros países, muchos casos se han gestionado por parte de la iglesia entregando grandes sumas de dinero a las víctimas por su silencio (Gavrielides, 2012). 
Esta dificultad de acceder a los sobrevivientes repercute en la limitación principal del presente estudio, esto es el tamaño de su muestra, lo cual a su vez condiciona el tipo de análisis estadísticos factibles de ser desarrollados y la generalización de los resultados obtenidos.

Junto con esto, el tipo de muestreo utilizado presenta restricciones respecto a la representatividad de los resultados, por cuanto la muestra no ha sido obtenida mediante métodos probabilísticos, y los participantes pueden ser aquellas víctimas más motivadas a responder, ya sea por uno u otro motivo. Esto se debe a que, dada la naturaleza del fenómeno estudiado, se carece de un marco muestral del cual obtener la muestra. Ello impide ofrecer iguales oportunidades de integrar la muestra a todos los participantes potenciales.

Concordantemente con lo anterior, la muestra obtenida no pretende ser representativa de la población total. De hecho, es posible apreciar en ella que la presencia de mujeres es muy superior a la encontrada en los estudios previos (John Jay College of Criminal Justice, 2004; Langeland et al., 2015), si bien otros estudios alertan de que las víctimas de sexo femenino quizás no se encuentren tan visibilizadas y, por tanto, pueda existir en los estudios previos un sesgo que debe tenerse en cuenta (Van Wormer y Berns, 2004). Del mismo modo, los sobrevivientes que participaron en la presente investigación poseen un nivel de estudios particularmente alto, siendo en su gran mayoría universitarios o postgraduados, lo cual puede haber influido en su decisión de colaborar en el estudio y, por tanto, en los resultados obtenidos.

A pesar de estas condicionantes, los resultados que se muestran permiten contar por primera vez con información muy valiosa respecto a las características del fenómeno en Chile, aspecto que constituye el aporte esencial de la presente investigación, siendo este un estudio pionero en Chile y en toda América Latina, hasta donde tenemos conocimiento.

Así, los resultados obtenidos permiten caracterizar a las víctimas de abuso sexual eclesiástico mayoritariamente como postpubescentes, y a sus abusadores como efebofílicos (Cimbolic y Cartor, 2006), lo cual se condice con la evidencia obtenida por la investigación del John Jay College of Criminal Justice (2004).

Tal como refieren otros investigadores (Rassenhofer et al., 2015), el abuso sexual eclesiástico suele ser de carácter reiterado, lo cual también se evidencia en esta investigación, pese a que su promedio de duración es inferior al encontrado en otras investigaciones (Lueger-Schuster et al., 2014). La gran mayoría de los sobrevivientes han 


\section{Contreras Taibo, Maffioletti Celedón, \& Pereda Beltrán}

padecido abusos muy intrusivos, fenómeno descrito por la literatura internacional (Denney et al., 2018; Rassenhofer et al., 2015); contemplando contacto corporal (tocaciones, masturbación, entre otros) y/o introducción de objetos o partes del cuerpo del victimario en el cuerpo de la víctima.

En este contexto, la religión que la víctima profesa y sus símbolos (cáliz, altar, crucifijo, sagrario) fueron utilizados para cometer el abuso. Se ha encontrado que el uso de símbolos, objetos o imágenes religiosas, o las creencias de la víctima, para cometer los abusos sexuales, es una característica específica de esta forma de victimización que puede conducir a un profundo daño espiritual (Isely, Isely, Freiburger y McMackin, 2008). Los sobrevivientes dan cuenta de alusiones directas del perpetrador a Dios y su voluntad para justificar estos delitos sexuales, refiriendo también el empleo de técnicas de neutralización que responsabilizan a la propia víctima de los abusos cometidos, en virtud de su condición de "pecador/a". La misma técnica de neutralización, denominada negación de la víctima, ha sido identificada por Spraitz y Bowen (2016) en sacerdotes acusados por delitos sexuales. Este proceso culpabilizador llevado adelante por el agresor, se puede ver facilitado por lo que Fairbairn (Rodriguez Sutil, 2010, p. 75) denomina defensa moral, mecanismo defensivo por el cual el niño incorpora en parte la maldad del objeto externo negativo, evitando así que este objeto idealizado y su contexto se constituyan en un entorno completamente maligno que le resulte insoportablemente amenazante. Por lo tanto, la recuperación de las víctimas no se puede limitar sólo a mitigar la sintomatología física o psicológica, sino que también se debe atender a los desafíos espirituales que implican este tipo de victimizaciones (Guido, 2008).

Respecto a la figura del victimario, y tal como refieren estudios previos (Denney et al., 2018; Rassenhofer et al., 2015), la gran mayoría de los abusos fueron perpetrados por varones, y en el caso de víctimas masculinas la totalidad de estos. Esto se puede comprender en el Chile de la década de los ' 80 y '90, toda vez que los colegios religiosos segregaban por sexo, teniendo contacto los niños hombres únicamente con representantes de la iglesia varones. En la muestra estudiada, más de la mitad de los perpetradores eran sacerdotes o párrocos, el resto de ellos eran laicos, consagrados o bien desempeñaban otro rol al interior de la iglesia, los cuales actuaron en general de forma solitaria.

La revelación de estos hechos sucedió en 8 de cada 10 casos, sin embargo, debieron pasar en promedio 16 años para que los sobrevivientes estuvieran en condiciones de relatar lo ocurrido a alguna persona en la edad adulta, lo cual da cuenta del profundo impacto que este tipo de abuso provocó en sus víctimas. Se observa, en los resultados 
obtenidos, que la mayoría de las víctimas requirieron de un tiempo de reflexión y maduración personal antes de poder revelar lo sucedido, como ya se ha indicado en trabajos previos con víctimas de abuso sexual (Tamarit, Abad y Hernández-Hidalgo, 2015).

El destinatario más frecuente de la primera revelación fue alguno de sus padres, la pareja, o un amigo/a. No obstante, 1 de cada 10 víctimas efectúa esta primera revelación a un miembro de la iglesia. Entre quienes revelaron este abuso, sólo cerca de la mitad se siente creído en su relato, sin embargo, tres cuartas partes percibieron una respuesta de apoyo por parte del entorno.

La notificación a las autoridades se produjo en menos de la mitad de las víctimas, y la mayor parte de ellas lo hace precisamente ante las autoridades eclesiásticas. Pese a ello, y de acuerdo al reporte de los sobrevivientes, en ninguno de los casos la autoridad eclesiástica informada de estos abusos denunció los hechos ante representantes del sistema investigativo-penal, como se ha observado en otros países (Doyle, 2009). Tal como señalan Guerzoni y Graham (2015), la exención de la obligación de informar por parte del clero vigente hasta entonces pudo contribuir al silenciamiento de esta grave forma de victimización al interior de la Iglesia Católica. Así, la iglesia intenta manejar la develación al interior de la comunidad, reemplazando la intervención del sistema de justicia por aquella de carácter religioso (Tishelman y Fontes, 2017). A su vez, la ausencia de denuncia y la impunidad generada a través de ella pudo permitir que los abusos continuaran, tanto hacia la misma víctima como hacia otras.

En este punto, la literatura demuestra que el sistema de creencias de quien investiga es crucial, pues las personas católicas tienden a ser más protectoras con los imputados, y más escépticos respecto al denunciante, particularmente cuando se encuentran altamente identificados con la religión (Minto et al., 2016). Esto lleva a reflexionar respecto a la necesidad de generar sistemas independientes a la iglesia para la notificación de este tipo de hechos, los cuales puedan garantizar la imparcialidad y autonomía necesaria para avanzar en términos de justicia y reparación.

Además del abuso sexual por parte de representantes de la iglesia católica, más del $80 \%$ de los sobrevivientes refieren otras formas de victimización, ya sea física, psicológica, negligencia y/o violencia sexual, fenómeno aún más prevalente en mujeres. Las diferentes formas de victimización se presentaron, en términos generales, tanto antes, como durante y con posterioridad al abuso eclesiástico. Así, muchos de los participantes del estudio manifiestan haber sido polivíctimas (Finkelhor, Ormrod y Turner, 2007b), lo 
que supone presentar unas necesidades de tratamiento y atención muy específicas que requieren del trabajo de profesionales altamente formados en aspectos de trauma complejo (Ford, 2015).

Si se comparan estas cifras de victimización con la población general en Chile (Brown, 2018), es posible apreciar que el porcentaje de abuso sexual no eclesiástico en la muestra estudiada duplica la prevalencia vida del abuso sexual infantil en población general. Esto remite al fenómeno de la revictimización, pues la literatura especializada señala que el riesgo de una nueva victimización sexual para quienes ya han sido víctimas respecto a quienes no lo han sido a lo menos se duplica, pudiendo llegar a ser hasta 7 veces mayor (Barnes, Noll, Putnam y Trickett, 2009; Widom, Czaja y Dutton, 2008; Finkelhor, Ormrod y Turner, 2007a).

Este estudio inicial en temáticas de victimización sexual infantil en contexto eclesiástico en Chile permite iluminar la complejidad de las experiencias vividas por los y las sobrevivientes, de manos de un representante de una iglesia en la cual habían confiado su desarrollo espiritual. Esto demanda la generación de respuestas integrales y decididas, tendientes a prevenir, detectar y otorgar todo el apoyo que las víctimas puedan requerir, en el difícil camino de la recuperación física, psicológica y espiritual. 


\section{Referencias}

AMM (2013). Declaración de Helsinki de la AMM - Principios éticos para las investigaciones médicas en seres humanos. Recuperado de https://www.wma.net/es/policies-post/declaracion-de-helsinki-de-la-ammprincipios-eticos-para-las-investigaciones-medicas-en-seres-humanos/

Azócar, M., y Undurraga, B. (2005). Reforma Procesal Penal y Seguridad Ciudadana: análisis de cuatro indicadores. Recuperado de https://www.icso.cl/images/Paperss/decimocuarto.pdf

Barnes, J., Noll, J., Putnam, F., y Trickett, P. (2009). Sexual and physical revictimization among victims of severe childhood sexual abuse. Child Abuse \& Neglect, 33, 412-420. http://doi.org/10.1016/j.chiabu.2008.09.013

Barth, J., Bermetz, L., Heim, E., Trelle, S., y Tonia, T. (2013). The current prevalence of child sexual abuse worldwide: A systematic review and metaanalysis. International Journal Public Health, 58, 469-483. http://doi.org/10.1007/s00038-012-0426-1

Böhm, B., Zollner, H., Fegert, J. M., y Liebhardt, H. (2014). Child sexual abuse in the context of the Roman Catholic Church: A review of literature from 19812013. Journal of Child Sexual Abuse, 23, 635-656. http://doi.org/10.1080/10538712.2014.929607

Brown, M. (2018). Informe final análisis multivariable de estudio polivictimización. Santiago: Consejo Nacional de la Infancia.

Calkins, C., Fargo, J., Jeglic, E., y Terry, K. (2015). Blessed be the children: A casecontrol study of sexual abusers in the Catholic Church. Behavioral Sciences and the Law, 33, 580-594. http://doi.org/10.1002/BSL.2193

Cancino, H. (2001). La Iglesia Católica y su contribución a la reconstrucción de la democracia en Chile 1973-1989. Revista del CESLA. International Latin American Studies Review, 2, 40-62. Recuperado de https://www.revistadelcesla.com/index.php/revistadelcesla

Cimbolic, P., y Cartor, P. (2006). Looking at ephebophilia through the lens of cleric sexual abuse. Sexual Addiction \& Compulsivity, 13, 347-359. http://doi.org/10.1080/10720160601011257

Colegio de Psicólogos de Chile (1999). Código de Ética Profesional (2a Ed.). Recuperado de http://colegiopsicologos.cl/web_cpc/wpcontent/uploads/2014/10/CODIGO-DE-ETICA-PROFESIONALVIGENTE.pdf

Contreras, L., Huepe, D. y Navarrete, G. (2020). Magnitude and characteristics of revictimization through child sexual abuse in Chile. Legal and Criminological Psychology, 25, 33-46. http://doi.org/10.1111//crp.12158

Denney, A., Kerley, K., y Gross, N. (2018). Child Sexual abuse in protestant christian congregations: A descriptive analysis of offense and offender characteristics. Religions, 9(27). http://doi.org/10.3390/rel9010027

Doyle, T. P. (2009). The spiritual trauma experienced by victims of sexual abuse by catholic clergy. Pastoral Psychology, 58, 239-260. http://doi.org/10.1007/s11089-008-0187-1

Echeburúa, E., y Guerricaechevarría, C. (2008). Concepto, factores de riesgo y efectos psicopatológicos. En J. Sanmartin (Ed.), Violencia Contra Niños (pp. 87-112). Barcelona: Ariel. 
Finkelhor, D., Ormrod, R., y Turner, H. (2007a). Re-victimization patterns in a national longitudinal sample of children and youth. Child Abuse \& Neglect, 31, 479-502. http://doi.org/10.1016/j.c hiabu.2006.03.012

Finkelhor, D., Ormrod, R., y Turner, H. (2007b). Poly-victimization: A neglected component in child victimization. Child Abuse \& Neglect, 31(1), 7-26. http://doi.org/10.1016/j.chiabu.2006.06.008

Ford, J. D. (2015). Complex PTSD: research directions for nosology/assessment, treatment, and public health. European Journal of Psychotraumatology, 6(1), 27584. http://doi.org/10.3402/ejpt.v6.27584

Gavrielides, T. (2012). Clergy child sexual abuse and the restorative justice dialogue. Journal of Church and State, 55(4), 617-639. http://doi.org/10.1093/jcs/css041

Guerzoni, M., y Graham, H. (2015). Catholic Church responses to clergy-child sexual abuse and mandatory reporting exemptions in Victoria, Australia: A discursive critique. International Journal for Crime, Justice and Social Democracy, 4(4), 58-75. http://doi.org/10.5204/ijcjsd.v4i4.205

Guido, J. J. (2008). A unique betrayal: Clergy sexual abuse in the context of the catholic religious tradition. Journal of Child Sexual Abuse, 17(3-4), 255-269. http://doi.org/10.1080/10538710802329775

Isely, P. J., Isely, P., Freiburger, J., y McMackin, R. (2008). In their own voices: A qualitative study of men abused as children by Catholic clergy. Journal of Child Sexual Abuse, 17(3-4), 201-215. http://doi.org/10.1080/10538710802329668

John Jay College of Criminal Justice (2004). The nature and scope of sexual abuse of minors by catholic priests and deacons in United States 1950-2002.

Recuperado de https://www.bishopaccountability.org/reports/2004 0227 JohnJay revised/2004 0227 John J ay_Main_Report_Optimized.pdf

Langeland, W., Hoogendoorn, A., Mager, D., Smit, J., y Dajier, N. (2015). Childhood sexual abuse by representatives of the Roman Catholic Church: A prevalence estimate among the Dutch population. Child Abuse \& Neglect: The international Journal, 46, 67-77. http://doi.org/10.1016/j.chiabu.2015.04.009

Lueger-Schuster, B., Kantor, V., Weindl, D., Knefel, M., Moy, Y., Butollo, A., . . . Gluck, T. (2014). Institutional abuse of children in the Austrian Catholic Church: Types of abuse and impact on adult survivors' current mental health. Child Abuse \& Neglect: The international Journal, 38, 52-64. http://doi.org/10.1016/j.chiabu.2013.07.013

Madrid, A. (2015). $4^{\circ}$ Estudio de maltrato infantil en Chile. Análisis comparativo. Santiago: UNICEF.

McLaughlin, B. R. (1994). Devastated spirituality: The impact of clergy sexual abuse on the survivor's relationship with God and the Church. Sexual Addiction \& Compulsivity, 1(2), 145-158. http://doi.org/10.1080/10720169408400039

Ministerio del Interior (2013). Encuesta nacional de victimización por violencia intrafamiliar y delitos sexuales. Santiago, Chile: División de Seguridad Pública.

Minto, K., Hornsey, M., Gillespie, N., Healy, K., y Jetten, J. (2016). A social identity approach to understanding responses to child sexual abuse allegations. PLOS ONE, 11(4), 1-15. http://doi.org/10.1371/journal.pone.0153205 
Pereda, N., Guilera, G., Forns, M., y Gómez-Benito, J. (2009). The prevalence of child sexual abuse in community and student samples: A meta-analysis.

Clinical Psychology Review, 29, 328-338. http://doi.org/10.1016/j.cpr.2009.02.007

Rassenhofer, M., Zimmer, A., Spröber, N., y Fegert, J. (2015). Child sexual abuse in the Roman Catholic Church in Germany: Comparison of victim-impact data collected through church-sponsored and government-sponsored programs. Child Abuse \& Neglect, 40, 60-67. http://doi.org/10.1016/i.chiabu.2014.11.013

Rodriguez Sutil, C. (2010). Introducción a la obra de Ronald Fairbairn: los origenes del psicoanalisis relaciona. Madrid: Ágora Relacional

Spraitz, D., y Bowen, K. (2016). Techniques of neutralization and persistent sexual abuse by clergy: A content analysis of priest personnel files from the Archdiocese of Milwaukee. Journal of Interpersonal Violence, 31(15), 1-24. http://doi.org/10.1177/0886260515579509

Stoltenborgh, M., van IJzendoorn, M., Euser, E., y Bakermans-Kranenburg, M. (2011). A global perspective on child sexual abuse: Meta-analysis of prevalence around the world. Child Maltreatment, 16(2), 79-101. http://doi.org/10.1177/1077559511403920

Tamarit, J.M., Abad, J., y Hernández-Hidalgo, P. (2015). Las víctimas de abuso sexual infantil ante el sistema de justicia penal: estudio sobre sus actitudes, necesidades y experiencia. Revista de Victimología, 2, 27-54. http://doi.org/10.12827-RVJV-2-02

Terry, K. J., Smith, M. L., Schuth, K., Kelly, J., Vollman, B., y Massey, C. (2011) Causes and context of the sexual abuse crisis in the Catholic Church. Washington DC: United States Conference of Catholic Bishops.

Terry, K. (2015). Child sexual abuse within the Catholic Church: A review of global perspective. International Journal of Comparative and Applied Criminal Justice, 39(2), 139-154. http://doi.org/10.1080/01924036.2015.1012703

Tishelman, A., y Fontes, L. (2017). Religion in child sexual abuse forensic interviews. Child Abuse \& Neglect, 63, 120-130. http://doi.org/10.1016/j.chiabu.2016.11.025

Van Wormer, K., y Berns, L. (2004). The impact of priest sexual abuse: Female survivors' narratives. Affilia, 19(1), 53-67. http://doi.org/10.1177/0886109903260667

Varona, G., y Martínez, A. (2015). Estudio exploratorio sobre los abusos sexuales en la Iglesia española y otros contextos institucionales: marco teórico y metodológico de una investigación victimológica abierta. Eguzkilore, 29, 776.

Widom, C., Czaja, S., y Dutton, M. A. (2008). Childhood victimization and lifetime revictimization. Child Abuse \& Neglect, 32, 785-796. http://doi.org/10.1016/j.chiabu.2007.12.006 